\title{
MICROQUASARS AND ULXS: FOSSILS OF GRB SOURCES
}

\author{
I. F. Mirabel ${ }^{1,2}$ \\ RESUMEN
}

Las fuentes de destellos gamma de larga duración probablemente resultan del colapso de estrellas masivas en sistemas binarios. Después del colapso de la estrella primaria el sistema binario puede permanecer ligado gravitacionalmente dejando como remanente un microcuásar o una fuente Ultraluminosa en rayos X (ULX). En este contexto, los microcuásares y las ULXs son fósiles de las fuentes de destellos gamma y contienen claves sobre la física y astrofísica de las fuentes de destellos gamma progenitoras. Aquí muestro que la identificación del lugar de nacimiento de los microcuásares acota las propiedades de las estrellas progenitoras de objetos compactos, y que la velocidad con que es disparado el microcuásar puede ser usada para acotar la energía de la explosión de las estrellas masivas que son progenitoras de estrellas de neutrones y agujeros negros. Las observaciones muestran que los sistemas binarios con estrellas de neutrones LS 5039, LSI +61 303 y el agujero negro de baja masa en GRO J1655-40 se formaron ('1 explosiones energéticas, mientras que los agujeros negros de masas mayores $\left(\mathrm{M} \geq 10 \mathrm{M}_{\odot}\right.$ ) en Cygnus X-1 y GRS $1915+105$ se formaron por colapso directo en oscuridad completa o en supernovas de baja luminosidad. La asociación con cúmulos de estrellas masivas de las estrellas de neutrones en LSI +61303 y de los magnetares SGR 1806-20 and SGR 1900+14, sugiere que estrellas muy masivas $\left(\mathrm{M} \geq 50 \mathrm{M}_{\odot}\right)$ pueden bajo ciertas circunstancias, terminar como estrellas de neutrones y no como agujeros negros. Lss fuentes de destellos gamma de larga duración contienen los mismos ingredientes básicos que los microcuásares y las ULXs, objetos compactos con discos de acreción y chorros relativistas en sistemas binarios, y las analogias fenomenológicas encontradas entre los microcuásares y Núcleos Activos de Galaxias podrán ser extendidas a las fuentes de destellos gamma.

\section{ABSTRACT}

Gamma-ray bursts (GRBs) of long duration probably result from the core-collapse of massive stars in binary systems. After the collapse of the primary star the binary system may remain bound leaving a microquasar or ULX source as remnant. In this context, microquasars and ULXs are fossils of GRB sources and should contain physical and astrophysical clues on their GRB-source progenitors. Here I show that the identification of the birth place of microquasars can provide constrains on the progenitor stars of compact objects, and that the runaway velocity can be used to constrain the energy in the explosion of massive stars that leave neutron stars and black holes. The observations show that the neutron star binaries LS 5039, LSI $+61^{\circ} 303$ and the low-mass black hole GRO J1655-40 formed in energetic supernova explosions, whereas the black holes of larger masses $(\mathrm{M} \geq 10 \mathrm{M} .0$ ) in Cygnus $\mathrm{X}-1$ and GRS $1915+105$ formed promptly, in the dark or in underluminous supernovac. The association with clusters of massive stars of the microquasar LSI $+61^{\circ} 303$ and the magnetars SGR 1806-20 and SGR 1900+11, suggest that very massive stars ( $M \geq 50 M_{\odot}$ ) may -in some cases- leave neutron stars rather than black holes. The models of GRB sources of long duration have the same basic ingredients as microquaisars and ULXs: compact objects with accretion disks and relativistic jets in binary systems. Therefore, the analogies between microquasars and AGN may be extended to the sources of GRBs.

\section{Key Words: ACCRETION, ACCRETION DISCS - X-RAYS: BINARIES - X-RAYS: STARS}

\section{MICROQUASARS AND GRB SOURCES}

Microquasars are black hole and neutron star binary systems that mimic, on smaller scale, many of the phenomena seen in quasars (Mirabel \& Rodríguez, 1999). In these sources accretion disks and jets are always coupled.

GRBs of long duration may be caused by the

\footnotetext{
'Service d'Astrophysique, CEA-Saclay, France.

${ }^{2}$ IAFE/CONICE.T. Argentina. fmirabel@cea.fr
}

formation of a black hole (collapsar; MacFayden \& Woosley, 1999) or a highly magnetized neutron star (Vietri \& Stella, 1998). It is estimated that half of the compact objects are produced in primordial binaries and that after their formation a significant fraction ( $20 \%)$ remain in binary systems (Belczynski et al., 2004), leaving microquasars as remnants.

It is believed that GRBs take place in close massive binaries because: 
1) the core must be spun up by spin-orbit interaction in order to provide enough power to the jet that will drill the collapsing star all the way from the core up to the external layers (Izzard et al., 2004, Podsiadlowski, et al., 2004).

2) GRBs seem to be associated to SNe Ic. This is the class of $\mathrm{SNe}$ that do not show $\mathrm{H}$ and He lines, implying that before the explosion the progenitor of those GRBs had lost the $\mathrm{H}$ and He layers. These layers are more easily lost if the progenitor was part of a massive binary that underwent a common envelope phase (Izzard et al., 2004, Podsiadlowski, et al., 2004). Furthermore, SNe Ic exhibit 4-7 \% polarization, which are an indication of asymmetric explosions caused by collimated jets.

\section{MICROQUASAR KINEMATICS AND THE CORE-COLLAPSE}

It is believed that stellar black holes can be formed in two different ways: Either the massive star collapses directly into a black hole without an energetic supernora explosion, or an explosion occurs in a protoneutron star, but the energy is too low to completely unbind the stellar envelope, and a large fraction of it falls back onto the short-lived neutron star, leading to the delayed formation of a black hole (Fryer et al., 2002). If the collapsar takes place in a binary that remains bound, and the core collapse produced an energetic supernova, it will impart the center of mass of the system with a runaway velocity, no matter the explosion being symmetric or asymmetric. Therefore, the kinematics of microquasars can be used to constrain theoretical models on the explosion of massive stars that form black holes.

Using this method it has been shown that the $\mathrm{x}$ ray binary Cygnux X-1 was formed in situ and did not receive an energetic trigger from a nearby supernova (Mirabel \& Rodrigues, 2003). If the progenitor of the black hole and its parent association Cygnus OB3 are coeval, the progenitor mass was greater than $40 \mathrm{M}_{\odot}$, and during the collapse to form the $10 \mathrm{M}_{\odot}$ black hole of Cygnus X-1, the upper limit for the mass that could have been suddenly ejected is $\sim 1 \mathrm{M}_{\odot}$, much less than the mass ejected in a typical supernova. Theoretical models suggest that larger mass remnants are associated to subluminous supernovae (Balberg \& Shapiro, 2001; Fryer et al, 2002).

Furthermore, the kinematics of GRS $1915+105$ derived from VLBA proper motion of the compact jet for the last 5 years (Dhawan \& Mirabel, 2004) show that the $14 \pm 4 \mathrm{M}_{\odot}$ black hole in this x-ray binary probably was formed promptly, as the black hole in Cygnus X-1. Of course, these observations do not exclude the possibility that high mass stellar black holes could also be formed with strong natal kicks, and runaway as unbound solitary black holes. which would be difficult to detect.

On the other hand, the kinematics of GRO J1655-40 has been a confirmation (Mirabel et al. 2002) of the indirect evidence for an energetic supernova explosion in the formation of this low-mass black hole, inferred earlier from the chemical composition of the donnor star (Israelian et al., 1999). More recently, the observation of the runaway x-ray binaries LS 5039 (Ribó et al., 2002) and LSI +61 303 (Mirabel et al., 2004) confirmed that neutron stars are formed with strong natal kicks.

\section{PROGENITORS OF NEUTRON STARS}

It is commonly believed that massive stars with masses above and below a fix mass limit leave black holes and neutron stars, respectively. However, the destiny of a massive star depends on several other factors besides on the mass, such as the metallicity and whether it is solitary or in a binary.

More massive stars evolve to the supernova stage faster, and a lower limit for the mass of the progenitor of the compact object can be determined assuming that the progenitor was coeval with the stars of the parent cluster.

It has been found that the neutron star $\mathrm{x}$-ray binary LSI $+61^{\circ} 303$ was shot out from its birth place in the cluster of massive stars IC 1805 and that the progenitor of the compact object had a mass $\geq 56$ $\mathrm{M}_{\odot}$ (Mirabel et al., 2004). However, this cluster is in a complex region and a lower mass progenitor cannot be ruled out since sequential star formation could have taken place in that region.

The location of magnetars (soft gamma-ray repeaters) in clusters of massive stars also sugest that neutron stars may have very massive stellar progenitors. Two of the four known magnetars (SGR 180620 and SGR 1900+14) are inside dust-enshrouded clusters of massive stars (Mirabel et al., 2000).

\section{ACCRETION-JET CONNECTION}

GRBs of long duration are believed to be produced in the accretion/ejection phenomena that take place in the formation of compact objects. Although the physical condition is different, the basic components in the collapsar model for GRB sources (MacFayden \& Woosley , 1999) and in the microquasar model shown in Figure 1 are analogous. Both models invoke accretion disks, relativistic jets, and binary systems. Therefore, the insight gained into the connection between accretion disk instabilities and the 


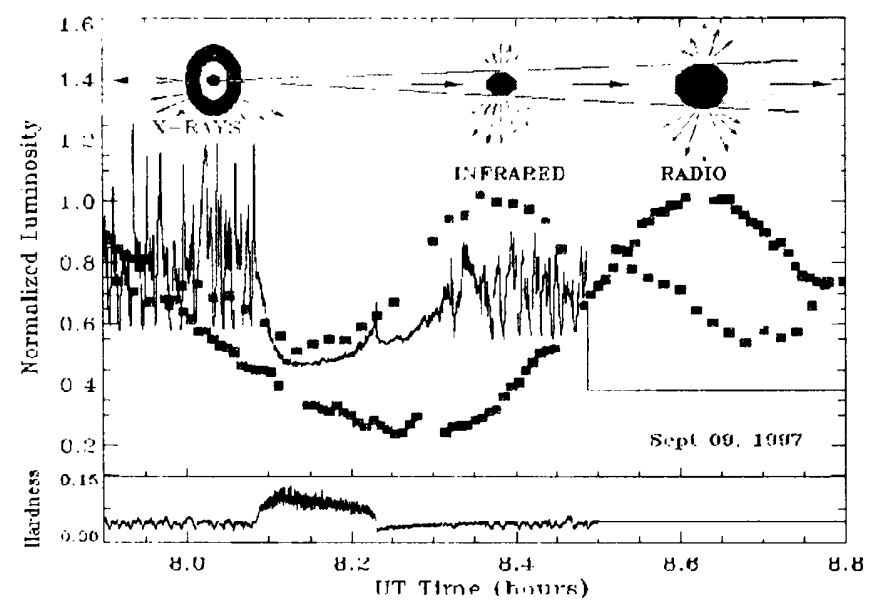

Fig. 1. Direst evidence for the disk-jet connection in the black hole x-ray binary GRS $1915+105$ (Mirabel et al. 1998). When the hot inner accretion disk disappeared, its $x$-ray brightness abruptly diminished. The rlsuing x-ray recovery documented the inner disk's replenishmenl. while the rising infrared and radio emission showed platsma being ejectorl in a jet-forming episode. The sequence of events shows that matcrial indeed was transfered from the disk to the jets. Similar transitions have becn olserved in the quasar 3C 120 (Marscher et al. 2002 ), but in time scales of ycurs, rather than minutes.

formation of jets in microquasars may be useful for our understanding of GRB sources. as has been the case for quasars (Marscher ot al. 20(0)2).

In $\sim 1$ hour of simultaneous multiwavelength observations of GRS' $1915+105$ during the frequently observed 30-to min $x$-riy oscillations in this source. the comnection between sudden drops of the x-ray flux from the acretion lisk and flir onset of jets were observed in several occasions (Mirabel et al., 1998. Eikenberry et al. 1998). Figure 1 shows simultaneous ofservations of this source in the x-rays, infrared. and radio wavelengths. From these observations we have kaned the following:

a) the jets appear after the drop of the x-ray flux, b) the jets are produced during the replenishment of the inner accretion disk.

c) the jet injertion is not instantancous. It can last up to $\sim 10 \mathrm{~min}$,

d) the time delay botween the jet flares at wavelengths of $2 \mu \mathrm{m}, 2 \mathrm{~cm} .3 .6 \mathrm{~cm}, 6 \mathrm{~cm}$, and $21 \mathrm{~cm}$ are consistent with the model of adiabatically expanding clouds that had been proposed to account for relativistic jets in AGN (van der Laan, 1966).

e) synchrotron emission is observed up to the infrared and probably up to $x$-rays. This would imply the presence in the jots of $\mathrm{TeV}$ electrons.

f) VLBA images during this type of x-ray oscillations
(Dhawan et al., 2000) showed that the ejecta consist on compact collimated jets with lengths of $\sim 100$ AU. g) there is a time delay of $\sim 5 \mathrm{~min}$ between the large drop of the x-ray flux from the accretion disk and the onsct of the jets. These $\sim 5$ silent minutes suggest that the compact object in GRS 1915+105 has a space-time border, rather than a material border, namely, a horizon as in relativistic black holes. However, this absence of evidence for a material surface may have alternative explanations.

After the observation of this accretion disk-jet connection in a microquasar, an analogous connection was observed in the quasar 3C 120 (Marscher et al., 2002), but in scales of years rather than minutes. This time scale ratio is comparable to the mass ratio between the supermassive black hole in 3C 120 and the stellar black hole in GRS 1915+105, as expected in the context of the black hole analogy.

Although GRBs are catastrophic events that take place only once and do not repeat as the outbursts in compact binaries, in both classes of sources the accretion/ejection phenomenology in stellar-mass black holes are invoked. An analogolis sequence of events to that shown in Figure 1 so far has not been observed in a GRB source.

\section{ULXS AND MICROQUASARS}

GRS $1915+105$ and SS 433 have been invoked as the Milky Way counterparts of the two classes of most numerous super-Eddington x-ray sources (ULXs) in external galaxies (King et al., 2002). GRS $1915+105$ is a long lasting transient outbursting x-ray binary with an evolved donnor of $\sim 1 \mathrm{M}_{\odot}$, whereas SS 433 is a persistent black hole HMXB (Hillwing et al. 2004). SS 433 type of ULX's are preponderantly found in starburst galaxies like the Antennac, whereas ULXs with low mass donors as GRS $1915+105$ may also be found in early type galaxies with a low rate of star formation.

Most of the ULX's would be stellar-mass black hole microquasars with the following possible properties: 1) HMXBs with stellar black holes of $M=20-40 M_{\odot}$ ) and isotropic radiation (Pakull \& Mirioni, 2003).

2) HMIXBs and LMXBs with stellar black holes (M $\sim 10 \mathrm{M}$ ) and anisotropic radiation (King, 2002).

3) A few $(\leq 1 \%)$ may be microquasars with relativistic boosted radiation. These should be very bright, highly time-variable, and have a hard $\mathrm{x}$-ray $/ \gamma$-ray photon spectrum (Mirabel \& Rodríguez, 1999).

Although much less numerous, it is not excluded that some ULX's could be accreting black holes of intermediate-mass (100-1000 $\mathrm{N}$ ). 


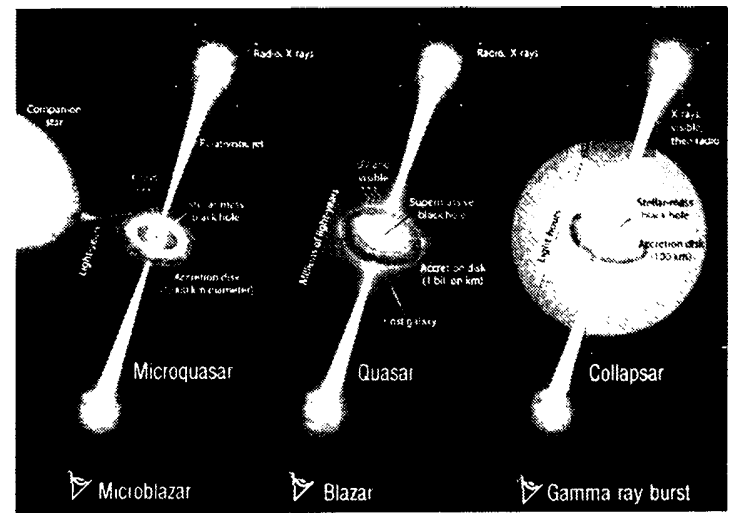

Fig. 2. A unique universal mechanism may be responsible for three types of astronomical objects: microquasars (left); quasars (center); and collapsars (right), the massive, suddenly collapsing stars believed to cause some gamma-ray bursts. Each contains a black hole (probably spinning), an accretion disk (which transfers material to the black holes), and relativistic jets (which emerge from a region just outside the black holes, carring away angular momentum). Microquasars and quasars can eject mattrer many times, while collapsars form jets but once. When the jet is aligned with an observer's line of sight these objects appear as microblazars, blazars, and gamma-ray bursters, respectively. The components of each panel are not drawn to scale; scale bars denote jet lengths. (Mirabel \& Rodríguez, 2002)

\section{JETS IN MICROQUASARS, AGN AND GRB}

As for AGN, it has been proposed (Lamb et al. 2004, Kouveliotou et al. 2004) a unification scheme where GRBs, x-ray flashes and SNe Ic are the same phenomenon, but viewed from different angles. However, radio observations of SNe Ic suggest (Soderberg et al., 2004) that the characteristics of supernovae are not dictated by the viewing angle but rather by the properties of the central engine. The observed variety of cosmic explosions (GRBs, x-ray flashes, $\mathrm{SNe}$ Ic) would then be explained by the varying fraction of the explosion energy that is channeled into relativistic ejecta.

It was suggested that irrespective of their mass there may be a unique universal mechanism for relativistic jets in accreting black holes (Mirabel \& Rodríguez, 2002; see Figure 2). Although in AGN, microquasars and GRB sources there are different physical conditions, it was proposed that all jets are produced by an unique electromagnetic mechanism, in which charged plasma is accelerated by electric fields that are generated by a rotating magnetic field (Meier, 2003). However, the most popular GRB jet models at this time are baryon dominated, and the factors that control the jet power. collimation and speed. remain unknown.

For this review I have gredty benefited from work done in collaboration with L.F. Rodríguez, I. Rodrigues, M. Ribó. S. Chatr. J. Martí. V. Dhawan. R. Mignani, D. Woi. J. Combi and L. Pellizza.

\section{REFERENC'ES}

Balberg, S. \& Shapiro. S.T. 2001, Ap.J 556. 914

Belczynski, Ki. Sadowski. A.. Rasio, F..1. 2004, astro$\mathrm{ph} / 0404068$

Dhawan V., Mirabel I.F., Rodríguez L.F., 2000, Ap.J 543. 373.

Dhawan, V. \& Mirabel, I.F. 2004, in preparation.

Eikenberry S.S.. Matthews K., Morgan F.H., et al. 1998. ApJ Letters 494, L61

Fryer C.L., Heger A., Langer N., and Vollstein S.. 2002. ApJ, 578, 335

Hillwing, T.C. et al. 2004, astro-ph/0403634

Israelian, G.; Rebolo, R.; Basri, G.; Cistares. J.; Martín, E. L. 1999, Nature, 401, 67.19. 142

Izzard R.G., Ramirez-Ruiz E., Tout C.A., 2004, MNRAS. 348, 1215

King, A.R. 2002, MNRAS 335, L13

Kouveliotou C., Woosley S. E., Patel S. K.. et al. 2004. astro-ph/0401184

Lamb D.Q, Donaghy T.Q. and Graziani C'., 2004, Neu.t $R$ 48, 459

MacFayden, A.I. \& Woosley, S.E., 1999, ApJ 524. 262.

Marscher A.P., Jorstad S.G. Gomez J.L. et al. $2002 \mathrm{Na}$ ture 417, 625

Meier, D.L. 2003, astro-ph/0312047

Mirabel I.F., Dhawan V., Chaty S., Rodríguez L.F. et al. 1998, $A \& A$ 330, L9

Mirabel, I. F.; Fuchs, Y.; Chaty. S. 2000. astroph/99912446 Published in Gamma-ray Bursts. 5th Huntsville Symposium. AIP Conference Series. $\mathrm{Vol}$. 526, p.814

Mirabel I.F., Mignani R., Rodrigues I., Combi J.A.. et al. 2002, $A \& A$ 395, 595

Mirabel I.F. and Rodríguez L.F. 1999, ARAA 37, 409.

Mirabel I.F. and Rodrigues I., 2003, Science 300, 1119

Mirabel I.F., Rodrigues I. \& Liu Q.Z. 2004. A. . A. in press

Mirabel I.F. and Rodríguez L.F., 2002, Sky \& Tels sopu. May issue, p.32.

Pakull M.W. and Mirioni L., 2003, RevMext.4 15. 197

Podsiadlowski, Ph. et al. 2004, astro-ph/0403399

Ribó M., Paredes J.M. Romero G.E., 2002. A 954

Soderberg A.M., Frail D.A., Wieringa M.H., 2004, astro$\mathrm{ph} / 0402163$

van der Laan. H. 1966 Nature 211. 1131

Vietri, M. \& Stella, L. 1998, ApJ 507. L.15 